\title{
Vortex Dynamics in Networks of Josephson Junctions
}

\author{
Ulrich Eckern \\ Institut für Theorie der Kondensierten Materie \\ Universität Karlsruhe \\ Karlsruhe, Federal Republic of Germany
}

\section{Introduction}

Since the proposal by Josephson ${ }^{1}$ in 1962 that a tunnel Junction, i.e. two superconductors weakly coupled through an oxide barrier, should show a zero-voltage supercurrent due to the tunneling of Cooper pairs, this system has been studied with unending enthusiasm. In particular, initiated by Leggett ${ }^{2}$ the last decade has seen a remarkable activity in a field which is often called Quantum Mechanics of Macroscopic Variables; ${ }^{3}$ experiments on small Josephson junctions at low temperature are found to be in excellent agreement with theoretical predictions.

As a matter of introduction, recall that the relevant variable for a tunnel junction is the order parameter phase difference $\varphi$, whose classical dynamies is determined by the following equations:

$$
H \varphi=2 e V: C V+V / R+I_{J} \sin \varphi=I_{T},
$$

where $V, C, R, I_{J}$, and $I_{T}$ denote the voltage, the capacitance, the resistance, the critical current, and the external current, respectively. Altematively:

$$
M A-\eta \dot{\theta}=-\partial U / \partial \varphi
$$

Here the potential is given by

$$
U(\omega)=-E_{j} \cos \varphi-f \cdot \omega
$$

where $E_{J}=\hbar I_{J} / 2 e$ is called the Josephson energy, $f=\hbar I_{T} / 2 e, M=(\hbar / 2 e)^{2} C$, and $\eta_{0}=(\hbar / 2 \pi) \cdot R_{0} / R: R_{0}=\pi \hbar / 2 e^{2} \sim 6.5 k \Omega$ denotes the unit of resistance.

Leaving aside for a moment the dissipative term, it is clear that the above equation can be "derived" from the Hamiltonian

$$
\mathcal{H}_{0}=Q^{2} / 2 C+U(\infty)
$$


by imposing the commutation relation $[Q, \varphi]=-2 e \cdot i$, where $Q$ is the charge difference across the junction. Phenomena related to the fact that $Q$ and $\varphi$ have, in principle, to be considered operators and not classical variables, become experimentally accessible in small junctions and for low temperature. More precisely, define the charging energy $E_{C}=e^{2} / 2 C$ and the parameter $\delta$ by

$$
\delta^{-1} \sim\left\{(\Delta \varphi)^{2}\right\rangle \sim\left(E_{C} / E_{J}\right)^{1 / 2},
$$

which also appears as exponent in a typical WKB calculation; note that $E_{J}$ is the potential barrier, and $\hbar \omega_{0} \sim\left(E_{C} E_{J}\right)^{1 / 2}$ the characteristic frequency. In the sixties, typical parameters have been such that $\delta \sim 10^{6}$, which allowed indeed to treat $\varphi$ as a classical varible. However, very recently, ${ }^{5}$ (networks of) tunnel junctions have been deviced with $\delta \sim 1$; thus it can be expected and is observed - that quantum phenomena are very pronounced in such a system.

Of course, given a classical equation like (2), it is not possible to derive the correct quantum mechanics of $\varphi$. In particular, note that $R^{-1}$ is often a sum of two contributions, namely

$$
R^{-1}=R_{t}^{-1}+R_{\Omega}^{-1}
$$

where $R_{t}$ is the strongly temperature dependent resistance due to quasiparticle tunneling, and $R_{\Omega}$ denotes an external shunt resistor. In order of magnitude, one has

$$
R_{t}(T) \sim R_{N} \cdot \exp (\Delta / k T)
$$

where $R_{N}=R_{t}\left(T_{c}\right)$, and $\Delta$ is the magnitude of the superconducting gap, taking the two superconductors to be identical for simplicity. As it turns out, in the presence of dissipation, the theoretical formulation proceeds most conveniently within the path integral formulation. ${ }^{6}$ As a result, ${ }^{7,8}$ an effective action is derived which contains, besides a term which follows from $\mathcal{H}_{0}$, two additive contributions describing the shunt resistor ${ }^{7}$ and the quasiparticle tunneling, ${ }^{8}$ respectively. While the former leads to a quadratic form, it is found that the latter has an important $4 \pi$-periodicity, ${ }^{9}$ which reflects the fact that the microscopic process is the tunneling of single electrons, ${ }^{10}$ in contrast to the continuous change of the charge in the presence of an external shunt. Note also that $\dot{\varphi} / R_{t}$ is the low frequency limit of a more general expression, ${ }^{8}$ which incorporates the creation of cuasiparticles for frequencies $\omega>2 \Delta / \hbar$.

In the following chapters, I wish to discuss some aspects of the low temperature behavior of a two-dimensional network of Josephson junctions, mainly based on the appropriate generalization of the Hamiltonian (4). In particular, $\mathrm{I}$ wish to pursue the dual description in terms of vortices, which is known to be adeciuate in the classical limit. ${ }^{11}$ Most of the material to be presented is based on a recent publication with Albert Schmid, in which further details and references can be found. ${ }^{12}$

\section{Description of the Model}

I consider a system of weakly coupled superconducting grains which form a two-dimensional square lattice. The relevant variables are the phases $\left\{\varphi_{i}\right\}$, where $\vec{l}=\left(l_{x}, l_{y}\right)$ labels the lattice sites (the lattice constant is taken to be unity), and the corresponding charges $\left\{Q_{i}\right\}$ :

$$
\left[Q_{i}, \varphi_{\bar{j}}\right]=-2 e \cdot i \delta_{\bar{l}, \bar{p}}
$$


The Hamiltonian of the network is given by $(f=0)$

$$
\mathcal{H}_{0}=\frac{1}{2} \sum_{\vec{l}, \vec{l}} Q_{\vec{l}}\left(\mathcal{C}^{-1}\right)_{\bar{l}, \bar{l}} Q_{\vec{l}}-E_{J} \sum_{\vec{l}, \vec{\mu}} \cos \left(\varphi_{\vec{l}+\vec{\mu}}-\varphi_{\bar{l}}\right)
$$

where $\vec{\mu}=(1,0)$ and $(0,1) ; c$ denotes the capacitance matrix. Its Fourier transform in the long wave-length limit is given by

$$
\mathcal{C}(\vec{q})=c_{0}+C q^{2}
$$

where $c_{0}$ and $C$ denote the ground and nearest-neighbor capacitance, respectively. In passing, I remark that $C$ is the sum of a geometric $\left(C_{0}\right)$ and a quasiparticle part, ${ }^{8}$ namely

$$
C=C_{0}+C_{q p}, C_{q p}=3 \alpha e^{2} / 16 \Delta,
$$

where I defined $\alpha=R_{0} / R_{N}$ as a dimensionless measure of the coupling between the grains (which is not a measure of the dissipation in the junctions). For example, $E_{J}=\alpha \Delta / 2$ in the low temperature limit. For distances large compared to the lattice spacing, the Coulomb interaction in real space $(r=|\vec{l}-\vec{l}|)$ follows from (10) to be given by

$$
\mathcal{C}^{-1}(r)=(2 \dot{\pi} C)^{-1} \cdot K_{0}(r / \lambda),
$$

which identifies $\lambda=\left(C / c_{0}\right)^{1 / 2}$ as the screening length. Thus peculiar features can be expected in the limit $c_{0}=0$, which means in particular $\lambda \gg N$, where $N$ is the size of the system (assuming $N^{2}$ grains). For the junctions described in Ref. 5, however, $\lambda$ is presumably of the order one, i.e. the Coulomb interaction is of short range.

From the Hamiltonian (9), the corresponding Euclidean action $S_{0}$ is derived in the usual way. ${ }^{6}$ Due to the extended nature of the vortices to be studied below, especially in the (uantum regime, it is sufficient (for some purposes) to consider the continuum limit, which is defined by the approximation

$$
\varphi_{\bar{l}+\bar{\mu}}-\varphi_{\bar{l}} \simeq(\vec{\mu} \cdot \nabla) \varphi_{\vec{l}} .
$$

Henceforth, I replace $\vec{l}$ by $\vec{r}$. Defining in addition $m=\hbar^{2} c_{0} / 4 e^{2}$, the action is given by

$$
S_{0}=\frac{1}{2} \int d \tau \int d^{2} r\left[m \dot{\varphi}^{2}+M(\nabla \dot{\varphi})^{2}+E_{J}(\nabla \varphi)^{2}\right] .
$$

Note that for finite temperature, the $r$-integration is restricted to $0 \ldots \hbar 3$, $\beta=(k T)^{-1}$, with periodic boundary conditions (see also Refs. 9 and 10). In addition, the external current contribution is

$$
S_{T}=-\int d \tau \int d^{2} r(\vec{f} \cdot \nabla) \varphi
$$

Finally, the dissipative contribution corresponds to a term in the action which is nonlocal in time; note that in the continuum limit, the special features of the quasiparticle part are not apparent. The result is

$$
S_{D}=\frac{1}{8} \int d \tau d \tau^{\prime} \int d^{2} r . A_{s}\left(\tau-\tau^{\prime}\right)\left[\nabla \varphi(\vec{r}, \tau)-\nabla \varphi\left(\vec{r}, \tau^{\prime}\right)\right]^{2} .
$$


Here I also neglected an unimportant contribution; ${ }^{12} A_{*}\left(\tau-\tau^{\prime}\right)$ is given by

$$
A_{s}\left(\tau-\tau^{\prime}\right)=\frac{\hbar \alpha_{s}}{\pi^{2}} \frac{1}{\left(\tau-\tau^{\prime}\right)^{2}}
$$

with $\alpha_{s}=R_{0} / R$ (recall that $\eta_{s}=(\hbar / 2 \pi) \alpha_{s}$ ). The above expressions apply in the continuum limit, in the adiabatic limit of small frequencies ( $\hbar \omega \ll 2 \Delta$ ), and for low temperature. The total action

$$
S=S_{0}+S_{T}+S_{D}
$$

is the basis of the following considerations (except for some final remarks).

Ignoring vortices for a moment, it is obvious that the response and the fluctuations of the system are characterized by the quantity

$$
\begin{aligned}
D_{\bar{q}, \omega}^{-1} & =\left[D_{0}^{-1}+\frac{1}{2} A_{0} \nabla^{2}\right]_{\vec{q}, \omega} \\
& =m \omega^{2}+\left[M \omega^{2}+E_{J}+\eta_{,}|\omega|\right] q^{2},
\end{aligned}
$$

from which the "spin-wave" dispersion $\omega_{\bar{q}}$,

$$
\omega_{\vec{q}}^{2}=\frac{(c q)^{2}}{1+(c q)^{2} / \omega_{0}^{2}},
$$

is easily identified. Here I defined $c^{2}=E_{J} / m$ and $\left(\hbar \omega_{0}\right)^{2}=8 E_{J} E_{C}$; note that $\lambda=c / \omega_{0}$, and that $\omega_{\vec{q}}=\omega_{0}$ for $m=0(\lambda=\infty)$.

\section{Statics and Dynamics of Vortices}

The transformation to the vortex picture is achieved by separating from $\varphi(\vec{r}, \tau)$ a contribution which incorporates explicitly the vortex configuration. Thus I put

$$
\varphi=\varphi^{v}+\varphi^{s}
$$

and choose $\varphi^{V}$ to be the appropriate solution of $\nabla^{2} \varphi^{V}=0$, namely

$$
\varphi^{V}(\vec{r}, \tau)=\sum_{j} e_{j} \arctan \frac{y-y_{j}(\tau)}{x-x_{j}(\tau)},
$$

where $\vec{r}_{j}(\tau)=\left(x_{j}(\tau), y_{j}(\tau)\right)$ is the center of the $j$ th vortex. Note that $\varphi^{V}$ is a multiple-valued function, which increases by $2 \pi e_{j}\left(e_{j}= \pm 1\right)$ as one goes around the $j$ th vortex; $e_{j}$ will also be called the charge of the vortex, and I impose at once the charge neutrality condition, $\Sigma_{j} e_{j}=0$. Inserting the ansatz (21) into the equation of motion, $D^{-1} \varphi=0$, I obtain for the spin-wave part $\varphi^{S}$ the following linear inhomogeneous equation:

$$
D^{-1} \varphi^{s}=\left(m-M \nabla^{2}\right) \partial_{r}^{2} \varphi^{V}
$$

which leads to

$$
\varphi_{\bar{q}, \omega}^{s}=-i \omega\left(m+M q^{2}\right) D_{\bar{q}, \omega}\left[\partial_{r} \varphi^{v}\right]_{\bar{q}, \omega}
$$


Inserting the total $\varphi$ into (18) leads to an effective action in terms of the vortex coordinates $\left\{\vec{r}_{j}\right\}$, which I denote by $\mathcal{A}$ (and $\mathcal{A}_{0}$, etc.). The following relations are useful:

$$
\begin{gathered}
{\left[\nabla \varphi^{V}\right]_{\vec{q}}=2 \pi i \sum_{j} e_{j} \frac{\left(q_{y},-q_{x}\right)}{q^{2}} e^{-i \bar{q} \cdot \vec{r}_{j}}} \\
{\left[\partial_{r} \varphi^{V}\right]_{\vec{q}}=2 \pi i \sum_{j} e_{j} \frac{q_{x} \dot{y}_{j}-q_{y} \dot{x}_{j}}{q^{2}} e^{-i \bar{q} \cdot \vec{r}_{j}} .}
\end{gathered}
$$

In addition, it is clear that in some expressions a large wave-vector cutoff is necessary, for example, as in the following quantity (see below):

$$
V(\vec{r})=E_{J} \int \frac{d^{2} q}{q^{2}}\left(e^{i \bar{q} \cdot \vec{r}}-1\right) e^{-q / q_{c}},
$$

where $q_{\mathrm{c}}=(2 \pi)^{1 / 2}$ as required by the original lattice model. Correspondingly,

$$
\int d^{2} q \rightarrow \int d^{2} q e^{-q / q_{c}}=2 \pi q_{c}^{2}=(2 \pi)^{2} .
$$

For the sake of transparency, I present the main features by concentrating on simple cases.

(i) $m=0(\lambda=\infty)$

In this case, in which the spin-wave spectrum has only an optical part, it is possible to neglect $\varphi^{S}$. With $\varphi \simeq \varphi^{V}$, it is straightforward to derive the following results:

$$
\begin{gathered}
A_{0}=\frac{1}{2} \int d \tau\left[\mathcal{M} \sum_{j} \dot{\vec{r}}_{j}^{2}+\sum_{i, j} e_{i} e_{j} V\left(\vec{r}_{i}-\vec{r}_{j}\right)\right] \\
\mathcal{A}_{T}=-2 \pi \sum_{j} e_{j} \int d \tau\left(f_{y} x_{j}-f_{x} y_{j}\right) \\
\mathcal{A}_{D}=\sum_{i, j} e_{i} e_{j} \int d \tau d \tau^{\prime} A,\left(\tau-\tau^{\prime}\right) F\left(\vec{r}_{i}(\tau)-\vec{r}_{j}\left(\tau^{\prime}\right)\right)
\end{gathered}
$$

Here the vortex mass is given by $\mathcal{M}=2 \pi^{2} M, V(\vec{r})$ is the (logarithmically increasing) interaction potential familiar from the classical limit, ${ }^{11}$ and $I$ have taken the external current, represented by $\vec{f}=\left(f_{x}, f_{y}\right)$, to be space independent. Furthermore, $F(\vec{r})$ is given by

$$
F(\vec{r})=-\frac{V(\vec{r})}{4 E_{J}}=\frac{\pi}{2} \ln \frac{1+\left(1+2 \pi r^{2}\right)^{1 / 2}}{2} .
$$

Ignoring the dissipative term $\mathcal{A}_{D},(29)$ and (30) correspond to the following equation of motion:

$$
\mathcal{M} \ddot{\bar{r}}_{i}=\overrightarrow{\mathcal{F}}_{i}-2 \pi e_{i} \hat{z} \times \vec{f},
$$


where $\hat{z}=(0,0,1)$, and $\overrightarrow{\mathcal{F}}_{i}$, representing the force due to the other vortices, is of the standard form: ${ }^{25}$

$$
\overrightarrow{\mathcal{F}}_{i}=-e_{i} \sum_{j \neq i} e_{j}(\nabla V)_{\vec{r}_{i}-\vec{r}_{j}}=-2 \pi E_{J} e_{i} \hat{z} \times\left(\nabla \varphi^{V}\right)^{\prime} .
$$

Here the prime, $\left(\nabla \varphi^{V}\right)^{t}$, indicates that the ith term has to be omitted in (22); also, I used the large distance result, $V(\bar{r}) \simeq-2 \pi E_{J} \ln r$, to arrive at the last equality. Note that a vortex moves perpendicular to the total current (external plus the current due to the other vortices).

In order to derive the dissipative contribution to (33), analytic continuation procedures are required.$^{8}$ Here I give only the final result, which follows from (31), namely

$$
M \ddot{\vec{r}}_{i}+2 e_{i} \sum_{j} e_{j} \int d t^{\prime} A_{s}^{R}\left(t-t^{\prime}\right)(\nabla F)_{\bar{r}_{i}(t)-\vec{r}_{j}\left(t^{\prime}\right)}=\ldots,
$$

where $A_{s}^{R}\left(t-t^{\prime}\right)$ is the Fourier transform of $A_{s}^{R}(\omega)=A_{s}(\omega \rightarrow-i \omega+0)$. Retaining only the term $i=j$, and in actdition expanding for small distances, I find

$$
\mathcal{M} \ddot{\vec{r}}_{i}+\tilde{\eta} \dot{\vec{r}}_{i}=\ldots \text {, }
$$

where $\bar{\eta}$ is given $b_{y} \vec{\eta}=2 \pi^{2} \eta s=\pi \hbar a$, which is not unexpected in view of the result $M=2 \pi^{2} M$.

(ii) $M=0, \eta_{s}=0$

In this case, it is rather cumbersome to follow the above procedure. flowever, it is olsvious from (14) that the problem is symmetric with respect to space and time (for zero temperature), which allows for the following elegant argument. $^{15}$ Delining $\overline{\vec{x}}=(x, y, c \tau)$, the corresponding gradient, $\bar{\nabla}$, and the "magnetic field" $\overline{\vec{h}}=\bar{\nabla} p$, the action (14) can be written as follows:

$$
S_{0}=\frac{E_{J}}{2 c} \int ; i^{3} x(\overline{\vec{h}})^{2} .
$$

Aso, introduce the charge and the current density by

$$
\rho=\sum_{j} c_{j} \delta\left(\vec{r}-\vec{r}_{j}\right), \quad \vec{j}=\sum_{j} e_{j} \dot{\vec{r}}_{j} \delta\left(\vec{r}-\ddot{r}_{j}\right) .
$$

Thus the continuity equation $\partial_{+} \rho+\nabla \cdot \vec{j}=0$ transforms into $\bar{\nabla} \cdot \overrightarrow{\vec{j}}=0$, where $\vec{j}=(\rho, \vec{j} / c)$. Finally, it is obvious that

$$
D^{-1} \varphi=0 \rightarrow \tilde{\nabla} \cdot \overline{\vec{h}}=0
$$

Choosing $\overline{\vec{h}}$ such that $\vec{\nabla} \times \overline{\vec{h}}=2 \pi \overline{\vec{j}}$, it follows from the analogy with the magnetostatic problem that $A_{0}$ is given by

$$
A_{0}=\frac{\pi E_{J}}{2 c} \int d^{3} x d^{3} x^{\prime} \frac{\overline{\vec{j}}(\overline{\vec{x}}) \cdot \overline{\bar{j}}\left(\tilde{\vec{x}}^{\prime}\right)}{\left|\overline{\vec{x}}-\overline{\vec{x}^{\prime}}\right|} .
$$


In terms of the vortex coordinates, this result reads

$$
\mathcal{A}_{0}=\frac{\pi E_{J}}{2 c} \sum_{i, j} \int d \tau d \tau^{\prime} \frac{\dot{\vec{r}}_{i}(\tau) \cdot \dot{\vec{r}}_{j}\left(\tau^{\prime}\right)+c^{2}}{\left\{\left[\vec{r}_{i}(\tau)-\vec{r}_{j}\left(\tau^{\prime}\right)\right]^{2}+c^{2}\left(\tau-\tau^{\prime}\right)^{2}\right\}^{1 / 2}} .
$$

The expression (40) is particularly appealing since it is of the same form as the magnetostatic energy of unimodular line currents (in three dimensions) flowing along the trajectories of the vortex centers. I only remark that some progress in the description of the phase transition of this model, which is expected to be of the 3D-XY type, in terms of vortex loops has been made recently. ${ }^{17}$

Of course, for a static configuration, i.e. $\vec{r}_{j}(\tau)$ independent of $\tau$, one may integrate with respect to time and recover the potential given in (29). More interesting, however, is the fact that (41) also includes a contribution which represents dissipation in the vortex motion due to decay into spin-waves. In particular, taking again the $i=j$ term and expanding for small velocities, $\left|\vec{r}_{j}(\tau)-\vec{r}_{j}\left(\tau^{\prime}\right)\right| \ll c\left|\tau-\tau^{\prime}\right|$, one obtains after Fourier transformation ${ }^{12,13}$

$$
\mathcal{A}_{0} \rightarrow \frac{1}{2} \sum_{j} \int \frac{d \omega}{2 \pi}|\omega| \eta(\omega)\left|\vec{r}_{j}(\omega)\right|^{2}
$$

where $\eta(\omega)=\pi m|\omega| \ln (1 /|\omega|)$ means a frequency dependent friction (which is called subohmic since $\eta(\omega) \rightarrow 0$ for $\omega \rightarrow 0$; this feature is connected with the fact that the vortices are coupled to a two-dimensional system of acoustic vibrations). It turns out, however, that the friction represented by $\eta(\omega)$ is weak in the sense that it leads only to logarithmic corrections to the free acceleration of a vortex by an external current. ${ }^{12}$

(iii) $M=0, \eta_{s} \neq 0$

I wish to remark that, in all cases, the evaluation of $\mathcal{A}$ can be simplified by the fact that $\left[\nabla \varphi^{S}\right]_{\vec{q}}$ is perpendicular to $\left[\nabla \varphi^{V}\right]_{\bar{\sigma}}$. Concerning the dissipation related to $\eta_{s}$, it follows from (19) that a characteristic frequency can be defined by

$$
\hbar \omega_{s}=\hbar E_{J} / \eta_{s}=2 \pi E_{J} / \alpha_{s}=\pi \Delta \alpha / \alpha_{s} .
$$

Assuming that $\alpha=R_{0} / R_{N} \gg \alpha_{9}$, it seems possible to expand the result (24) with respect to $\eta_{s}$. In particular, it follows that the expression $\dot{\varphi}^{2}+c^{2}\left(\nabla \varphi^{5}\right)^{2}$ has only a correction in second order. As a result, the contribution $\sim \eta_{\text {s }}$ to $\mathcal{A}$ is precisely given by $(31)$, and $\mathcal{A}_{0}$ retains the form given in (41) in this order.

Finally, I briefly discuss the case where both $M$ and $m$ are different from zero. As a rule, it seems that the acoustic vibrations implied by a finite value of $m$ dominate at large distances in space and time. This means that the most important consequence of a finite $M$ is the effective mass $\mathcal{M}$ of a vortex. Besides the kinetic energy term in (29), the effective action is thus given by (41), (30), and (31), at least as long as $\alpha_{s}$ is small. A particular case is the limit in which $\lambda=(M / m)^{1 / 2}$ is larger than the sample dimension. Then it is possible to put $m=0$ and take $\mathcal{A}_{0}$ as given by (29). Presumably, the last case is mostly of theoretical interest. 


\section{Vortex Dynamics in Other Systems}

Without going into great detail, I summarize in this section the classical equations of motion which are often used to describe vortex dynamics in superfluid ${ }^{4} \mathrm{Helium}$ and in superconductors. In order to simplify the notation, I denote the coordinate of the vortex under consideration by $\vec{r}_{0}(t)$, and define $\vec{v}_{\text {, }}$ to be the local superfluid velocity at $\vec{r}_{0}$, however, excluding the velocity field of this particular vortex. Also, $e_{0}= \pm 1$ is the sign of the vorticity and, having especially films in mind, $d$ is the film thickness, and $\rho$, the superfluid mass density integrated across the film; finally, $m^{*}$ denotes the Helium mass and twice the electron mass, respectively.

\section{(i) Superfluid Helium Films}

The equation of motion is obtained by balancing the Magnus force against the viscous drag due to interactions with thermal excitations and with the substrate (see Vinen, ${ }^{15}$ and also Ref. 18):

$$
B \dot{\vec{r}}_{0}+B^{\prime} e_{0} \bar{z} \times \dot{\vec{r}}_{0}=e_{0} \rho_{s} \frac{2 \pi \hbar}{m^{*}} \hat{z} \times\left(\dot{\vec{r}}_{0}-\vec{v}_{*}\right)
$$

where $B$ and $B^{\prime}$ are phenomenological coelficients. Of particular importance is the form of the rhs of (44), which leads to the conclusion that for $B=B^{\prime}=0$, the vortex rides along with the local superfluid velocity. It seems that the Magnus force as given in (44) is intimately related to the Galileil invariance of the Helium system, and the above conclusion is believed to hold at low temperature and for a transiation invariant substrate. Generally, the vortex moves in a direction characterized by the angle $\theta$ (defined such that $\theta=0$ if the vortex moves perpendicular to $\vec{v}_{\mathrm{s}}$ ), which is given by

$$
\tan \theta=\left(2 \pi \hbar \rho_{s} / m^{*}-B^{\prime}\right) / B
$$

\section{(ii) Superconducting Films}

The theory of vortex dynamics in superconductors has been first discussed in detail by Bardeen and Stephen, ${ }^{19}$ and is essentially based on the idea that a vortex has a normal core of radius $\sim \xi$, where $\xi$ is the coherence length. (For an overview, see Tinkham's book. ${ }^{20}$ ) Thus dissipation is due to ordinary Ohmic losses, i.c. through electrons scattering at static impuritics or defects, in the normal region. Their result reads: ${ }^{19}$

$$
\eta\left(\dot{\vec{r}}_{0}-\kappa_{H} \vec{v}_{s}\right)=\rho_{s} \frac{2 \pi \hbar}{m^{*}} \hat{z} \times\left(-\vec{v}_{s}\right),
$$

where I have taken $c_{0}=1$; the friction constant $\eta$ is given by

$$
\eta=\frac{R_{0}}{R_{N}} \frac{\hbar}{\xi^{2}}=R_{0} \sigma_{N} d \frac{\hbar}{\xi^{2}}
$$

with $\sigma_{N}$ the normal state (Drude) conductivity. In addition, $\kappa_{H}$ is given by

$$
\kappa_{H}=H / H_{c 2}, \quad H_{\mathrm{c} 2}=\phi_{0} / 2 \pi \xi^{2}
$$

where $H$ is the magnitude of the magnetic field (which is applied perpendicular to the film), and $\phi_{0}$ the flux quantum. (However, shortly thereafter it was 
argued $^{21}$ that $\kappa_{H}=1$ should be the correct result.) Note that $\eta$ is essentially independent of temperature.

Using that at low temperature the integrated superfluid density is given by $2 \rho_{s} / m^{*}=n d$, where $n$ is the total electron density, the Hall angle is found to be given by the same expression as in the normal state, namely

$$
\tan \theta=\omega_{c} \tau \sim \kappa_{H} \frac{\Delta}{\epsilon_{F}} \frac{\ell}{\xi},
$$

where $\omega_{e}$ is the cyclotron frequency, $\tau$ the elastic scattering time, $\ell=v_{F} \tau$ the mean free path, $\epsilon_{F}$ the Fermi energy, and I used that $\xi \sim \hbar v_{F} / \Delta$, which applies for $\ell \gg \xi$. Note that the Hall angle is very small, except for extremely pure samples, since $\Delta \sim 10^{-4} \epsilon_{F}$. It seems that the debate about the Hall angle was somehow inconclusive (see, however, Ref. 22), which also may be related to the fact that pinning of vortices by impurities ${ }^{23}$ - neglected above - plays experimentally a far more important role; ${ }^{20}$ on the other hand, the theory of the viscosity as well as of nonlinear phenomena is well developed. ${ }^{24}$

In comparison, I wish to emphasize what I believe is an important difference between Helium and superconductors, which is apparent from (44) and (46). In the former case, the vortex moves in the direction of the local $\vec{v}$, in the limit of vanishing viscosity, $B \rightarrow 0$, with a velocity smaller than $\vec{v}_{s}$ provided $B^{\prime}$ is finite. In the latter case, however, the limit of an extremely clean metal corresponds to a very strong viscosity, i. e. formally $\eta \rightarrow \infty$. In this limit, the vortex moves in the direction of the local $\vec{v}_{s}$, with a velocity smaller than $\vec{v}_{\text {, if }} \kappa_{H}<1$. It appears that the dynamics of vortices is fundamentally different in the two systems.

Finally returning to the model of a network of Josephson junctions studied in the preceding sections, I emphasize that I do not find any indication of a finite $\theta$. Rather, the vortices are found to move perpendicular to the local current, which seems somehow surprising since the viscosity is small at low temperature. Presently, I can only speculate that the underlying lattice structure (which means e.g. that the vortices do not have a normal core) is responsible for this result.

\section{Conclusion}

In these notes, I presented selected aspects of the statics and the dynamics of a network of Josephson junctions, within the framework of the dual description in terms of vortices. In this approach, it is straightforward to make contact with the classical (high temperature) limit results. ${ }^{11}$ I remark that the lattice model (for $M=0$ ) has been studied recently by extensive Monte Carlo simulations, which show a reentrant behavior upon decreasing the temperature, as well as a first order transition within the superconducting region. ${ }^{25}$ However, for example, the effect of a finite nearest-neighbor capacitance $(M \neq 0)$ on the phase diagram, especially at zero temperature, is still an open question. ${ }^{26,10}$ In addition, I wish to mention that recent experiments on granular ${ }^{27}$ as well as on continuous ${ }^{28}$ superconducting films have shown several unexpected features, pointing towards the normal state film resistance as the (most?) important parameter.

As a word of caution, I emphasize that I restricted myself to discuss the adiabatic limit of the general model, ${ }^{12}$ i.e. the limit of small frequencies 
$\hbar \omega \ll 2 \Delta$. In this case, the parameter $R_{N}$ or $\alpha=R_{0} / R_{N}$, where $R_{N}$ equals the normal state sheet resistance (for a square sample) in the absence of shunt resistors, enters only indirectly through $E_{J}$ and the quasiparticle capacitance. However, the adiabatic condition is, at best, only marginally satisfied when $E_{C}$ and $E_{J}$ are of the order of a few Kelvin. For example, for $\alpha_{s}=0$, a vortex is freely accelerated by an external current until its energy is large enough to create quasiparticles, ${ }^{12}$ i.e. is larger than $2 \Delta$. This suggests that for a detailed understanding of the dynamics at low temperatures, the full nonlinear model has to be considered, which unfortunately is a formidable problem. On the other hand, I emphasize that high frequencies probe the "normal" part of the current-voltage characteristic, which possibly may explain the significance of the normal state resistance.

\section{Acknowledgement}

Financial support by the Deutsche Forschungsgemeinschaft through a Heisenberg fellowship is gratefully ackrowledged.

\section{References}

1. B. D. Josephson, Phys. Letters 1, 251 (1962); Advan. Phys. 14, 419 (1965).

2. A. J. Leggett, Suppl. Progr. Theor. Phys. 69, 80 (1980).

3. For a recent review and further references, see: A. J. Leggett, Jap. J. Appl. Phys. 26, Suppl. 26-3, 1986.(1987):

4. P. W. Anderson, in Lectures on the Many Body Problem, edited by E. R. Caianiello (Academic, New York, 1964), Vol. 2, p. 113.

5. L. J. Geerligs, M. Peters, L. E. M. de Groot, A. Verbruggen, and J. E. Mooij, Phys. Rev. Lett. 63, 326 (1989).

6. R. P. Feynman and A. R. IHibbs, Quantum Mechanics and Path Integrals (McGraw-Hill, New York, 1965).

7. A. O. Caldeira and A. J. Leggett, Plyss. Rev. Lett. 46, 211 (1981); Ann. Phys. (N. Y.) 140, 374 (1083).

8. V. Ambegaokar, U. Eckern, and G. Schön, Phys. Rev. Lett. 48, 1745 (1982); Phys. Rev. B 30,-6419 (1984); A. I. Larkin and Yu. N. Orchinnikov, Phys. Rev. B 28, 6281 (1983).

9. F. Guinea and G. Schön, Europhys. Lett. 1, 585 (1986); J. Low Temp. Phys. 69, 219 (1987).

10. For a recent review see, for example: U. Eckern and G. Schön, in Festkörperprobleme/Advances in Solid State Physics, edited by U. Rössler (Vieweg, Braunschweig, 1989), Vol. 29, p. 1.

11. V. L. Berezinskii, Zh. Eksp. Teor. Fiz. 61, 1144 (1972) (Sov. Phys. JETP 34, $610(1972)$; J. M: Kosterlitz and D. J. Thouless, J. Phys. C 5, L124 (1972); 6, 1181 (1973).

12. U. Eckern and A. Schmid, Phys. Rev. B 30,6441 (1989); see also Ref. 13.

13. A. I. Larkin, Yu. N. Ovchinnikov, and A. Schmid, Physica B 152, 266 (1988).

14. Compare Eq. (2.4) of Ref. 12.

15. Y. B. Kim and M. J. Stephen, in Superconductivity, edited by R. D. Parks (Dekker, New York, 1969), Vol. 2, p. 1107; W. F. Vinen, ibid. p. 1167. 
16. V. N. Popov, Functional Integrals in Quantum Field Theory and Statistical Physics (Reidel, Boston, 1983), Sec. 21.

17. S. R. Shenoy, Phys. Rev. B (1989), to be published.

18. V. Ambegaokar, B. I. Halperin, D. R. Nelson, and E. D. Siggia, Phys. Rev. B' 21, 1806 (1980).

19. J. Bardeen and M. J. Stephen, Phys. Rev. 140, A1197 (1965).

20. M. Tinkham, Introduction to Superconductivity (McGraw-Hill, New York, 1975), Sec. 5.

21. P. Nozières and W. F. Vinen, Philos. Mag. 14, 667 (1966).

22. J. Bardeen and R. D. Sherman, Phys. Rev. B 12, 2634 (1975).

23. A. Schmid and W. Hauger, J. Low Temp. Phys. 11, 667 (1973).

24. See, for example: A. Schmid, Phys. Kondens. Materie 5, 302 (1966); A. I. Larkin and Yu. N. Ovchinnikov, Pis'ma Zh. Eksp. Teor. Fiz. 23, 210 (1976) [JETP Lett. 23, 187 (1976)]; Zh. Eksp. Teor. Fiz. 73, 299 (1977) [Sov. Phys. JETP 46, 155 (1977)].

25. L. Jacobs, J. V. José, M. A. Novotny, and A. M. Goldman, Phys. Rev. B 38, 4562 (1988); L. Jacobs and J. V. José, Physica B 152. 148 (1988); J. Choi and J. V. José, Phys. Rev. Lett. 62, 1904 (1989).

26. S. Chakravarty, S. Kivelson, G. T. Zimanyi, and B. I. Halperin, Phys. Rev. B 35, 7256 (1986); R. A. Ferrell and B. Mirhashem, ibid. 37, 648 (1988); S. E. Korshunov, Europhys. Lett. 0, 107 (1989); W. Zwerger, ibid. 9, 421 (1989).

27. B. G. Orr, H. M. Jaeger, A. M. Goldman, and C. G. Kuper, Phys. Rev. Lett. 56, 378 (1986); H. M. Jaeger, D. B. Haviland, A. M. Goldman. and B. G. Orr, Phys. Rev. B 34, 4920 (1986).

28. D. B. Haviland, Y. Liu, and A. M. Goldman. Phys. Rev. Lett. 62, 2180 (1989). 